archives

of thermodynamics

Vol. 36(2015), No. 4, 87-105

DOI: 10.1515 /aoter-2015-0035

\title{
Investigation of transient heat transfer in composite walls using carbon/epoxy composites as an example
}

\author{
JANUSZ TERPIEOWSKI ${ }^{a 1}$ \\ BARTOSZ GAWRON ${ }^{a}$ \\ GRZEGORZ WORONIAK ${ }^{b}$
}

a Military University of Technology, Kaliskiego 2, 00-908 Warsaw, Poland

${ }^{b}$ Bialystok Technical University, Wiejska 45A, 15-351 Bialystok, Poland

\begin{abstract}
The paper presents the application of similarity theory to investigations of transient heat transfer in materials with complex structure. It describes the theoretical-experimental method for identification and design of the structure of two-component composite walls based on the research of the thermal diffusivity for the composite and its matrix separately. The thermal diffusivity was measured by means of the modified flash method. The method was tested on two samples of double-layer 'epoxy resin - polyamide'. All the investigated samples had the same diameter of $12 \mathrm{~mm}$ and thickness ranging from $1.39-2.60 \mathrm{~mm}$ and their equivalent value of thermal diffusivity ranging from $(1.21-1.98) \times 10^{-7} \mathrm{~m}^{2} / \mathrm{s}$. Testing the method and research on carbon/epoxy composites was carried out at temperatures close to room temperature.
\end{abstract}

Keywords: Thermal properties; Flash method; Thermal diffusivity; Carbon/epoxy composites; Carbon fiber; Epoxy resin

\section{Nomenclature}

$$
\begin{array}{lll}
a=\lambda /\left(\rho c_{P}\right) & - & \text { thermal diffusivity, } \mathrm{m}^{2} / \mathrm{s} \\
c_{P} & - & \text { specific heat, } \mathrm{J} /(\mathrm{kg} \mathrm{K}) \\
l & - & \text { thickness of the investigated sample, } \mathrm{m}
\end{array}
$$

\footnotetext{
${ }^{1}$ Corresponding Author. E-mail: janusz.terpilowski@wat.edu.pl
} 


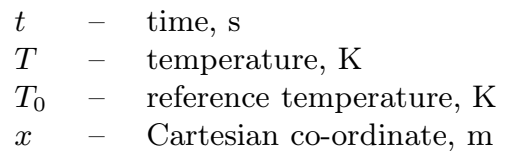

Greek symbols

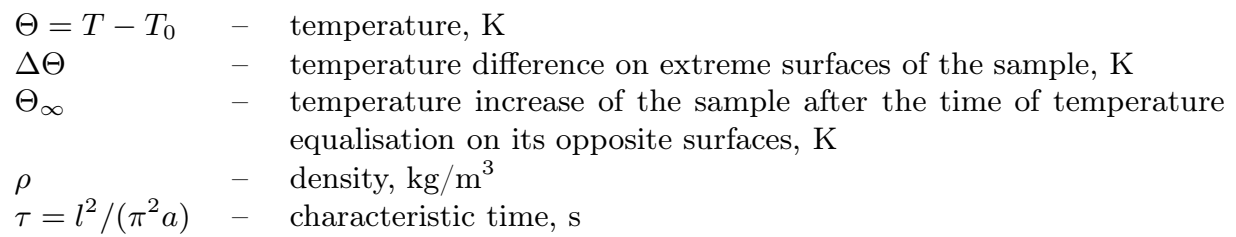

\section{Introduction}

The method of investigating transient heat transfer in a two-component composite wall presented in this paper is based on the model in which the heterogeneous structure of composite was replaced by equivalent layer of the matrix material. Therefore, in this case, a one-dimensional homogeneous model was used in the simulation of transient heat transfer. In accordance with the principles of the theory of similar phenomena $[1,2]$ as a basis for determining the equivalent thickness of the two-component composite in a simulation model with the properties of homogeneous material, equal values of Fourier numbers for the total thickness of matrix material and layers of the composite treated as reinforcement in the wall, were assumed. Further course of action, related to the construction and solution model transient heat transfer in plane-parallel isotropic wall, with the replacement thickness $l_{E, S}$, is identical with the model used to determine the thermal diffusivity by a modified pulse method [3-5].

Applying the principles of similarity phenomena theory in the study of transient heat transfer in composite walls, constructed of materials with different thermal diffusivity values, has the advantage that it binds together in the form of an additional equation (equations) the thermal diffusivity of the composite components, the equivalent thermal diffusivity and geometric dimensions of wall.

The consequence of the practical application of this research method is the ability to determine by calculation:

- the equivalent thermal diffusivity of the composite if known are the thermal diffusivities and thicknesses of layers of components from which the composite wall is built; 
- of known is thermal diffusivity value of one of the components of the composite in case when the equivalent thermal diffusivity of composite wall and thermal diffusivity of the second component and the thickness of the layers of each component in the wall are known.

\section{Assumptions and the transient heat transfer model}

The method of investigating transient heat transfer in two-component composites presented in this paper is based on the model in which the heterogeneous structure of composite, i.e., in the form of carbon fiber plate, was replaced by equivalent layers of the matrix material (epoxy resin). Therefore, in this case a one-dimensional homogeneous model was used in the simulation of transient heat transfer. A sample of the composite and its characteristic dimensions taken into account are shown in Fig. 1.

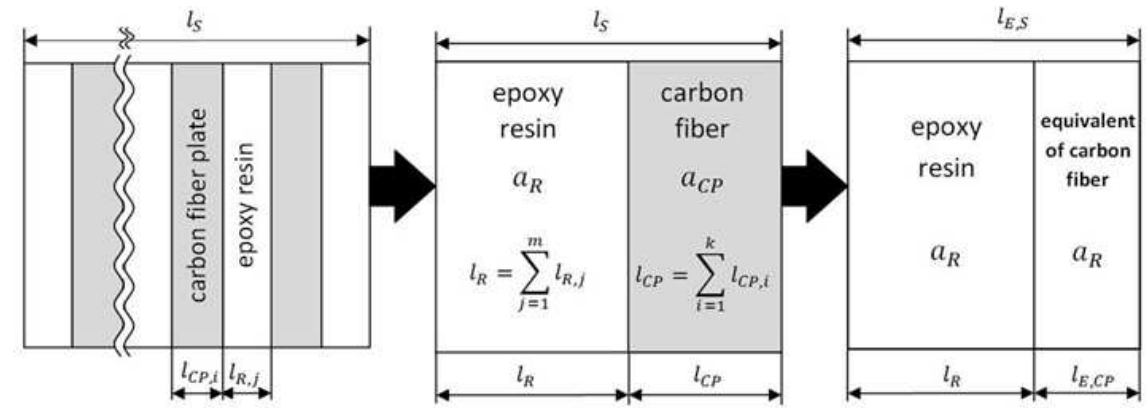

Figure 1: Characteristic dimensions of the composite sample: $l_{S}$ - thickness of the investigated sample; $l_{R}$ - total thickness of epoxy resin layers in the sample; $l_{C P}-$ total thickness of carbon fiber layers in the sample; $l_{E, S}-$ equivalent thickness of the homogeneous sample in the simulation model made of the matrix; $l_{E, C P}$ - equivalent thickness of the matrix material used in the simulation model instead of the carbon fiber layers with thickness $l_{C P} ; a_{R}$ and $a_{C P}$ - thermal diffusivity of epoxy resin and carbon fiber layer suitable.

In order to determine the equivalent value of the thermal diffusivity of composite material, which by definition is a material with anisotropic thermophysical properties, the following simplifying assumptions were made:

- layers of carbon fiber plate with the same thickness, in the form of braided carbon fiber inside epoxy resin, are located parallel to both 
the extreme surfaces of a flat-parallel, cylindrical sample and perpendicular to the heat flux flowing through the sample;

- thermal contact resistance between layers of epoxy resin and carbon fiber plate is equal to zero;

- the model of heat transfer in the composite material is one-dimensional and the temperature distribution inside the model is determined by solving the Fourier equation in the form:

$$
\frac{\partial^{2} \Theta(X, \mathrm{Fo})}{\partial X^{2}}=\frac{\partial \Theta(X, \mathrm{Fo})}{\partial \mathrm{Fo}},
$$

where

$$
\Theta=\Theta(X, \mathrm{Fo})=T(X, \mathrm{Fo})-T_{0}
$$

is the temperature difference between temperature $T\left(X, F_{O}\right)$ at non-dimensional $X=(x / l)$ coordinate and the initial constant temperature inside the sample $T_{0}=T\left(X, F_{O}=0\right)$;

$$
\mathrm{Fo}=a t / l^{2}
$$

is the Fourier number, it's dimensionless time coordinate, wherein: $a-$ thermal diffusivity; $t$ - time; $l$ - is the thickness of the sample.

Equations of transient heat transfer that determine one-dimensional temperature distributions in two different but homogeneous materials (Fig. 1), with the boundary conditions shown in Fig. 3, have the following forms:

$$
\frac{\partial^{2} \Theta_{1}}{\partial \mathrm{X}^{2}}=\frac{\partial \Theta_{1}}{\partial \mathrm{Fo}_{1}}, \frac{\partial^{2} \Theta_{2}}{\partial \mathrm{X}^{2}}=\frac{\partial \Theta_{2}}{\partial \mathrm{Fo}_{2}},
$$

where

$$
\mathrm{Fo}_{1}=\frac{a_{1} t_{1}}{l_{1}^{2}} \quad, \quad \mathrm{Fo}_{2}=\frac{a_{2} t_{2}}{l_{2}^{2}}
$$

If we assume that:

$$
\mathrm{Fo}_{1}=\mathrm{Fo}_{2}=\mathrm{Fo}
$$

and when thermal contact resistance between layers is equal to zero, then the course of temperature changes in both materials will be identical and determined by Eq. (1). The relationship between the Fourier number and the characteristic time $\tau=l^{2} /\left(\pi^{2} a\right)$ or the half-time $t_{0.5}=1.368 l^{2} /\left(\pi^{2} a\right)$, which are easy to determine experimentally (flash methods to determine thermal diffusivity), has the form:

$$
\text { Fo }=\pi^{-2}(t / \tau)=1.368 \pi^{-2}\left(t / t_{0.5}\right) .
$$


Thus, the assumption (3), after taking into account (4) can be reduced to the form: $t_{1} / \tau_{1}=t_{2} / \tau_{2}$. If additionally, the time scale of the course of temperature changes in both samples is the same, that is $t_{1}=t_{2}=t$, then the equality of the Fourier numbers is reduced to the assumption that characteristic times $\tau$ of temperature changes in the two considered samples (layers) are the same, that is

$$
\tau_{1}=\tau_{2}=\tau \text {. }
$$

In the case of two-component composite material, assuming that in the simulation model, the base material of the sample (matrix) is epoxy resin, the layer of carbon fiber plate with the total thickness $l_{2}=l_{C P}$ and the thermal diffusivity $a_{2}=a_{C P}$ is replaced by the equivalent layer of epoxy resin with the thermal diffusivity $a_{1}=a_{R}$ and thickness:

$$
l_{E, C P}=\left(a_{R} / a_{C P}\right)^{0.5} l_{C P} .
$$

Therefore, the sample of anisotropic composite (matrix of epoxy resin and $k$ layers of carbon fiber plate) with the $l_{S}$ thickness is equivalent in the simulation model to the sample of the matrix material (epoxy resin) with the thermal diffusivity $a_{R}$ and thickness:

$$
l_{E, S}=l_{R}+l_{E, C P}=l_{R}+\left(a_{R} / a_{C P}\right)^{0.5} l_{C P}=\pi\left(a_{R} \tau_{E, S}\right)^{0.5} .
$$

Taking into account the fact that it is easy to determine the experimental values of the characteristic times $\tau_{R}$ (for the sample built of the composite matrix) and $\tau_{E, S}$ (for the sample of the composite) and the corresponding values of $l_{R}, l_{E, S}$ and $a_{R}$ respectively, we can find a formula:

$$
\triangle l=l_{S}-l_{E, S}=l_{C, P}-l_{E, C P}
$$

for the thickness reduction (see Fig. 1) between the composite sample and its equivalent in the simulation model, built of the same material (the composite matrix). When the thermal diffusivity $a_{R}$ of the composite matrix as well as the experimentally determined thicknesses $l_{S}$ and $l_{E, S}$ of the investigated sample are known, taking into account the geometrical dependences (8), shown in Fig. 1, and dependence (7), we have:

$$
\frac{l_{C P}}{l_{S}}=\left(1-\frac{l_{E, S}}{l_{S}}\right)\left[1-\left(\frac{a_{R}}{a_{C P}}\right)^{0.5}\right]^{-1} .
$$




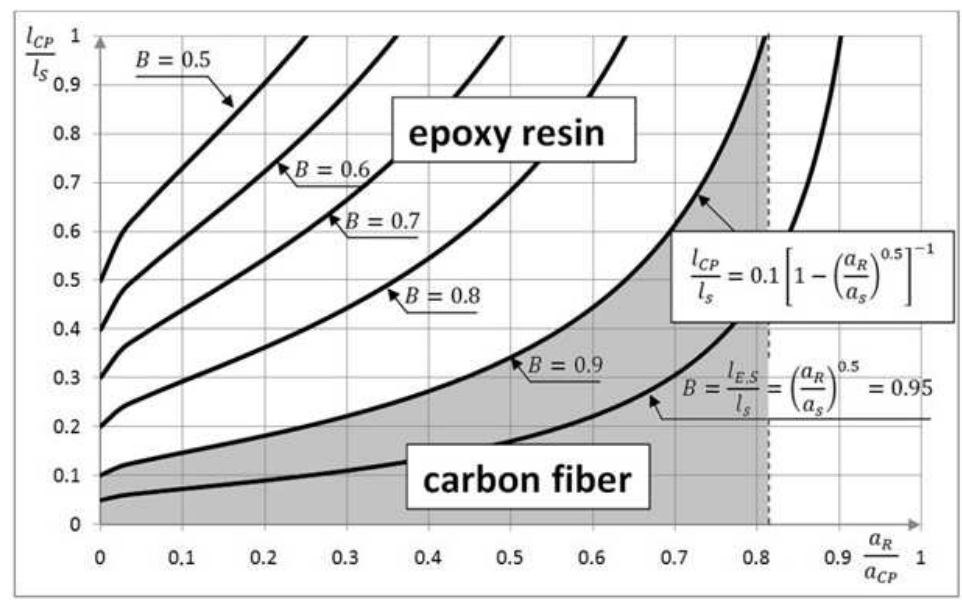

Figure 2: Graph of the function $\left(l_{C P} / l_{S}\right)_{B}=f\left(a_{R} / a_{C P}\right)$ for some selected values of $B=l_{E, S} / l_{S}$ and the theoretical example of a possibility of building a composite wall with the value $B=0.9$ and equivalent value of the thermal diffusivity $a_{S}=B^{2} a_{R}=1.234 a_{R}$.

The graph of the function (9) is shown in Fig. 2. It can be used both for the analysis and synthesis of the structure of multilayer walls designed for work in unsteady heat exchange.

In both instances we assume that the values of the matrix thermal diffusivity $a_{R}$ and the composite wall thickness $l_{S}$ and its equivalent thickness $l_{E, S}$ are known from independent measurements. Therefore we also know the value of the parameter:

$$
B=l_{E, S} / l_{S}=\left(a_{R} / a_{S}\right)^{0.5}
$$

and the equivalent value of the thermal diffusivity $a_{S}$ of the composite wall. The data and relation (9) and its graph shown in Fig. 2 allow us to evaluate the theoretical range of possibilities of designing a wall of composite with the above parameters. For example, if $B=l_{E, S} / l_{S}=0.9$, then such a wall whose thickness is $l_{S}$ and the thermal diffusivity $a_{S}=B^{2} a_{R}=1.234 a_{R}$ can be built of materials whose thicknesses and thermal diffusivities satisfy the equation:

$$
l_{C P} / l_{S}=0.1\left[1-\left(a_{R} / a_{C P}\right)^{0.5}\right]
$$

as shown in Fig. 2.

Figure 3 shows an example of temperature distribution (ABC curve) at time $t_{i}$, in the real multilayer sample (two-layer sample) with thickness $l_{S}$ 
between extreme surfaces of this sample. The distribution of temperature for this sample in the simulation model is given by ABD curve. The thickness $l_{R}$ of the resin layer remains unchanged. The temperature distribution in these layers of resin to be the same as that in the real sample. However, the layer of carbon fabric with thickness $l_{C P}$ is replaced by the equivalent thickness of resin $l_{E, C P}$ in the simulation model, wherein the excess temperature distribution will have a different course than in the layer of carbon fabric in real sample, but the temperature difference $\Delta \Theta\left(t_{i}\right)$ at the time $t_{i}$ on extreme surfaces of layers with thicknesses $l_{C P}$ and $l_{E, C P}$ will be the same. The sample created on the basis of resin will have a thickness $l_{E, S}$ in the simulation model.

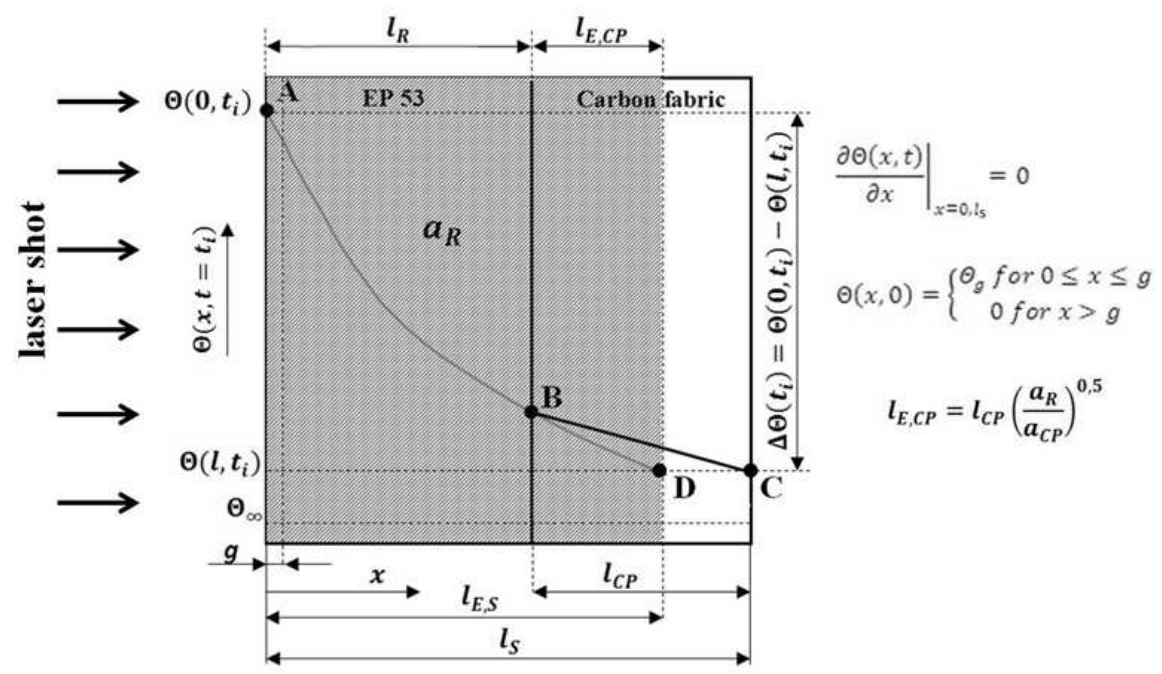

Figure 3: Example of temperature distribution $\Theta\left(x, t=t_{i}\right)$ in the multilayer sample and in the same sample based on resin EP53 in the simulation model with characteristic dimensions.

\section{Testing of the method and an example of its application to identifying the characteristic fea- tures of the carbon/epoxy composite structure}

To demonstrate the suitability of the above method for the identification of an unknown composite structure or for the design of a new one with set 
values of the equivalent thermal diffusivity, the method was tested and a robust example of its application was shown.

\subsection{The method for determining the characteristic time and thermal diffusivity a of investigated samples}

To test the method proposed to investigate the transient heat exchange in heterogeneous solids we applied the model described by Parker et. al. [6]. The modified pulse method [3,4] used in these investigations, and its essential elements are shown in Fig. 4. This method is based on:

- measurement of changes of temperature difference $\Delta \Theta^{\prime}(t)$ between the front and back surfaces of a one-dimensional and adiabatic sample, after a laser shot onto its front surface (Fig. 3);

- theoretical solution to this problem, in the form of changes of temperature difference $\triangle \Theta(t)$ on extreme surfaces of the sample made of a homogenous material, with thickness $l$ and the thermal diffusivity a. It is given by:

$$
\triangle \Theta(t)=4 \Theta_{\infty} \sum_{n=1}^{\infty} \exp \left[(2 n-1)^{2} \frac{t}{\tau}\right],
$$

where: $\Theta_{\infty}$ - increase of the sample temperature after the laser shot and after the end of the transient heat transfer process inside the adiabatic sample; $\tau=l^{2} /\left(\pi^{2} a\right)$ - characteristic time for the sample temperature equalization; $T_{0}$ - initial temperature of the sample (before a laser shot, see Fig. 4);

- identification of the best fitting of the experimental results $\triangle \Theta^{\prime}(t)$ by a theoretical curve $\triangle \Theta(t)$. The optimisation parameter is the characteristic time:

$$
\tau=\left(t_{2}-t_{1}\right)\left[\ln \frac{\Delta \Theta^{\prime}\left(t_{1}\right)}{\Delta \Theta^{\prime}\left(t_{2}\right)}\right]^{-1},
$$

which depends on the thermal diffusivity $a$. The value of the $\tau$ corresponding to the best fitting is regarded as a correct one.

When determining the equivalent characteristic time $\tau_{E}$ and the equivalent thermal diffusivity $a_{E}$ of the heterogeneous sample with thickness $l_{S}$, e.g., built of two layers with thickness $l_{1}$ and $l_{2}$ and with the thermal diffusivities 


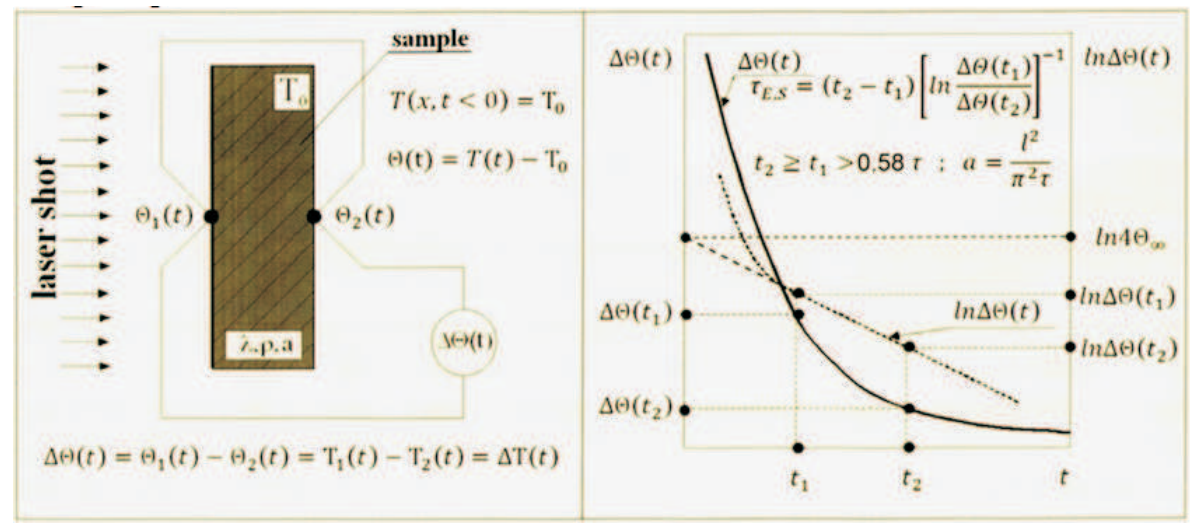

Figure 4: The modified pulse method of determining the characteristic time $t$ and the thermal diffusivity $a$ of a homogeneous material sample.

$a_{1}$ and $a_{2}$ the sample is treated as a homogeneous, and the temperature change $\Delta \Theta(t)$ on the opposite surfaces of the sample is given by

$$
\triangle \Theta(t)=4 \Theta_{E, \infty} \sum_{n=1}^{\infty} \exp \left[(2 n-1)^{2} \frac{t}{\tau_{E}}\right] .
$$

The errors of the thermal diffusivity $a$ measurement and the characteristic time $\tau$ by means of the above method have been estimated as $\triangle a / a<0.04$ and $\triangle \tau / \tau<0.02$ respectively (the values of errors were estimated during the modified flash method developing) [3-5].

\subsection{Method testing using epoxy/polyamide composite}

The main idea in this test was to check whether calculated using the proposed method in this work thermal diffusivity $a_{T}$ value of polyamide PA6 is consistent with the values known from independent measurements.

Testing the proposed method was performed on two-layer composite two samples 'epoxy resin EP53 - polyamide PA6'. All the parameters of the each composite sample, i.e., their thickness $l_{S, i}$ and the thermal diffusivities $a_{S, i}$, as well as thickness of the layers $l_{R, i}$ and $l_{T, i}$ for $i=1,2$ are known. The thermal diffusivities $a_{R}$ and $a_{T}$, suitable for EP53 resin and polyamide PA6 were also known from independent measurements that were made for samples with only one layer EP53 or PA6.

Examples of the final printouts of the experimental data processing during the determination of characteristic times $\tau_{E, S}$ and $\tau_{T}$, as well as the cor- 

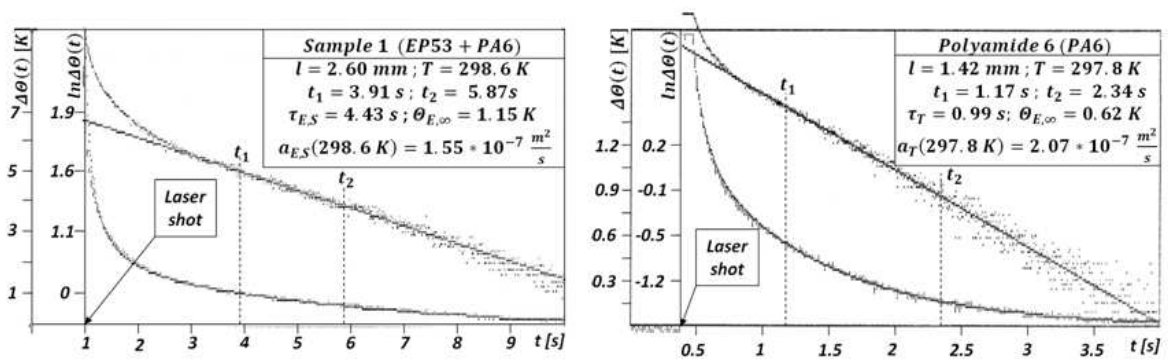

Figure 5: Distribution of experimental data processing during determination by the modified pulse method the $\tau_{E, S}$ and $a_{E, S}$ for the epoxy/polyamide PA6 composite (sample 1) and $\tau_{T}$ and $a_{T}$ for the polyamide PA6 sample.

responding values of thermal diffusivities $a_{E, S}$ and $a_{T}$ are shown in Fig. 5. When several composite samples with various thickness of layers $l_{R, i}$ and $l_{T, i}$ their thicknesses have to be standardized prior to further calculations. Here, the thickest sample was taken as a standard thickness $l_{S}^{S}$ for both samples. This means that for that thickest sample (sample 1), its thickness and its equivalent thickness are the same $\left(l_{S}^{S}=l_{S 1}\right.$ and $\left.l_{E, S 1}=l_{E, S 1}^{S}\right)$. In the case of the other sample (sample 2) we have $l_{S}^{S}$ and $l_{E, S 2}^{S}=l_{E, S 2}+\left(l_{S}^{S}-l_{S 2}\right)$. The overall dimensions and the thickness standardization final results are summarized in Tab. 1 and in Fig. 6.
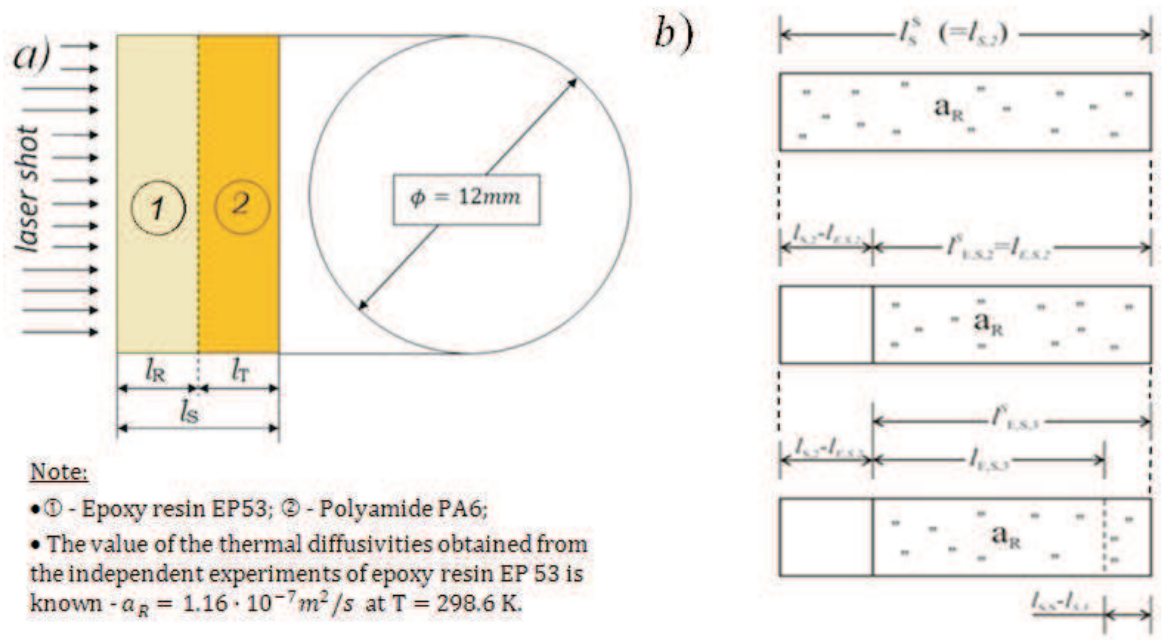

Figure 6: a) Geometry of composite samples; b) final result of the standardization of composite samples under investigation. 
Investigation of transient heat transfer in composite walls. .

Table 1: Dimensions of the composite samples, thermal diffusivities of its materials and results of testing method.

\begin{tabular}{|c|c|c|c|c|}
\hline \multirow{2}{*}{ Specification } & \multicolumn{2}{|c|}{ Sample 1} & \multicolumn{2}{|c|}{ Sample 2} \\
\hline & (1) - EP 53 & (2) - PA6 & (1) - EP 53 & (2) $-\mathrm{PA} 6$ \\
\hline Thickness of layers (1) and (2), [mm] & 1.10 & 1.50 & 1.30 & 1.00 \\
\hline Thickness of the sample, $l_{S, i}[\mathrm{~mm}]$ & \multicolumn{2}{|c|}{2.60} & \multicolumn{2}{|c|}{2.30} \\
\hline Characteristic time $\tau_{E, S i}[\mathrm{~s}]$ & \multicolumn{2}{|c|}{4.43} & \multicolumn{2}{|c|}{3.77} \\
\hline $\begin{array}{l}\text { Equivalent thickness of the sample, } \\
l_{E, S, i}[\mathrm{~mm}]\end{array}$ & \multicolumn{2}{|c|}{2.25} & \multicolumn{2}{|c|}{2.07} \\
\hline $\begin{array}{l}\text { Thickness of the sample after the } \\
\text { standardization, } l_{S, i}^{S}[\mathrm{~mm}]\end{array}$ & \multicolumn{2}{|c|}{2.60} & \multicolumn{2}{|c|}{2.60} \\
\hline $\begin{array}{l}\text { Equivalent thickness of the sample af- } \\
\text { ter standardization, } l_{E, S, i}^{S}[\mathrm{~mm}]\end{array}$ & \multicolumn{2}{|c|}{2.25} & \multicolumn{2}{|c|}{2.37} \\
\hline Parameter $B_{i}^{S}=\left(l_{E, S} / l_{S}\right)_{i}^{S}$ & \multicolumn{2}{|c|}{0.865} & \multicolumn{2}{|c|}{0.911} \\
\hline$\left(l_{T} / l_{S}\right)_{i}^{S}$ & \multicolumn{2}{|c|}{0.577} & \multicolumn{2}{|c|}{0.385} \\
\hline$\left(a_{R} / a_{T}\right)_{i}$ & \multicolumn{2}{|c|}{0.587} & \multicolumn{2}{|c|}{0.591} \\
\hline$a_{R}\left[\mathrm{~m}^{2} / \mathrm{s}\right]$ & \multicolumn{4}{|c|}{$1.16 \times 10^{-7}$} \\
\hline$a_{T, i}\left[\mathrm{~m}^{2} / \mathrm{s}\right]$ & \multicolumn{2}{|c|}{$1.98 \times 10^{-7}$} & \multicolumn{2}{|c|}{$1.96 \times 10^{-7}$} \\
\hline
\end{tabular}

Assuming that the thermal diffusivity of the epoxy $a_{R}$ is known, and the thickness of the samples $l_{S}$ and the thickness $l_{T}$ of the material imitating the reinforcement of the composite sample, we determined characteristic time $\tau_{E, S, 1}$ (for sample 1) and $\tau_{E, S, 2}$ (for sample 2), their equivalent thickness based on equation (7) are: $l_{E, S, 1}=2.25 \mathrm{~mm}$ and $l_{E, S, 2}=2.07 \mathrm{~mm}$ in the simulation model. Thus the determined values of the dimensionless parameters $B_{1}^{S}=\left(l_{E, S} / l_{S}\right)_{i=1}^{S}=0.865, B_{2}^{S}=\left(l_{E, S} / l_{S}\right)_{i=2}^{S}=0.911$, $\left(l_{T} / l_{S}\right)_{i=1}^{S}=0.577$ and $\left(l_{T} / l_{S}\right)_{i=2}^{S}=0.385$, which when substituted into (9) make it possible to determine the value of dimensionless thermal diffusivity $\left(a_{R} / a_{T}\right)_{i=1}=0.587$ and $\left(a_{R} / a_{T}\right)_{i=2}=0.591$ (Tab. 1, Fig. 7). Because the value of the thermal diffusivity of epoxy resin is known $a_{R}=1.16 \times 10^{-7} \mathrm{~m}^{2} / \mathrm{s}$ $($ at $T=298.6 \mathrm{~K})$, then $a_{T, 1}=1.98 \times 10^{-7} \mathrm{~m}^{2} / \mathrm{s}, a_{T, 2}=1.96 \times 10^{-7} \mathrm{~m}^{2} / \mathrm{s}$ and the obtained value of thermal diffusivity of the polyamide 6 (material) is similar to the value in Fig. 5 - in the range of error band for the modified pulse method.

Values of thermal diffusivity $a_{T, 1}$ and $a_{T, 2}$ of polyamide PA6 obtained using this method are in fact identical $a_{T} \cong(1,97 \pm 0,01) \times 10^{-7} \mathrm{~m}^{2} / \mathrm{s}$ (Tab. 1) and vary from the value $a_{T}=2.07 \times 10^{-7} \mathrm{~m}^{2} / \mathrm{s}$ given by the independent measurement (Fig. 5) by less than $5 \%$. 


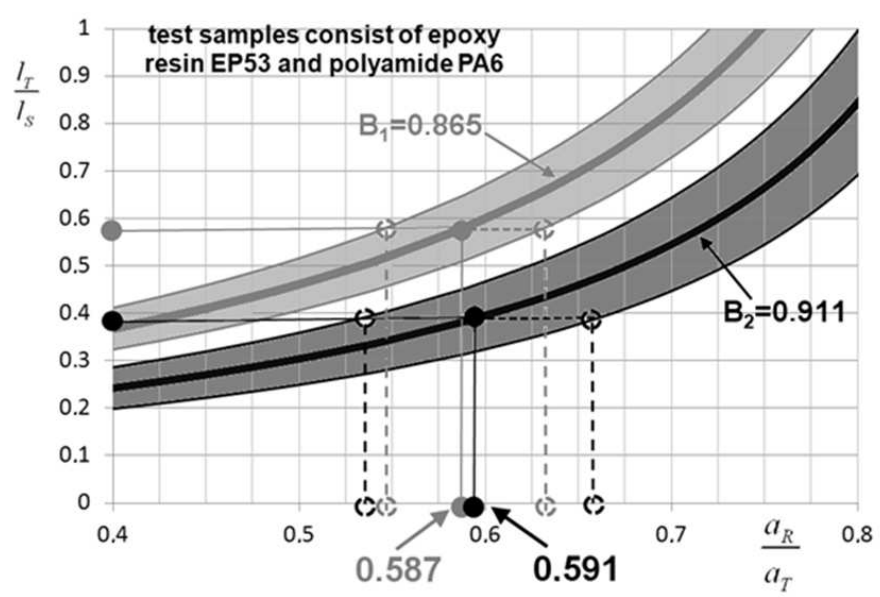

Figure 7: Results from testing the method for the epoxy/polyamide PA6 composite (see Tab. 1).

Metrological capacity of this method of studying transient heat transfer in composite walls depends on the estimation of errors made while determining two out of three values in $(9)$, that is $\left(l_{T} / l_{S}\right)_{i}^{S}, B_{i}^{S}=\left(l_{E, S} / l_{S}\right)_{i}^{S}$ and $\left(a_{R} / a_{T}\right)$. However, the error in determination of $B_{i}^{S}$ seems to be the principal component in the total error estimation process. For $l_{E, S}$ given by (7), i.e. $l_{E, S}=\pi\left(a_{R} \tau_{E, S}\right)^{0.5}$, the relative error of $B_{i}^{S}$ is:

$$
\delta_{B, i}=\left|\triangle B_{i}^{S} / B_{i}^{S}\right|=0.5\left[\left|\triangle a_{R} / a_{R}\right|+\left|\left(\triangle \tau_{E, S} / \tau_{E, S}\right)_{i}^{S}\right|+2\left|\triangle l_{S}^{S} / l_{S}^{S}\right|\right] .
$$

In the process of determining both thermal diffusivity $a_{R}$ and characteristic time $\tau_{E, S, i}$ using the modified pulse method, all the quantities in (14) are calculated by a special sub-program incorporated into the measurement data acquisition and processing program used at the test stand. In our case, for the samples under investigation we have: $\left|\triangle a_{R} / a_{R}\right|=0.022$, $\left|\left(\triangle \tau_{E, S} / \tau_{E, S}\right)_{i=1}^{S}\right|=0.002,\left|\left(\triangle \tau_{E, S} / \tau_{E, S}\right)_{i=2}^{S}\right|=0.003$ and $\left|\triangle l_{S}^{S} / l_{S}^{S}\right|=$ 0.004. After substituting these data into (14) we obtain $\delta_{B, 1}=0.016$ and $\delta_{B, 2}=0.016$. The error made while estimating $\left(l_{T} / l_{S}\right)_{i}^{S}$ is $\delta_{\triangle l / l, 1}=0.01$ and $\delta_{\triangle l / l, 2}=0.014$, respectively. Upon including all the calculated values of $\delta_{B, 1}, \delta_{B, 2}, \delta_{\triangle l / l, 1}$, and $\delta_{\triangle l / l, 2}$, for the dimensionless values of parameter $\left(l_{T} / l_{S}\right)_{1}^{S}=0.577$ and $\left(l_{T} / l_{S}\right)_{2}^{S}=0.385$ the dimensionless values of thermal 
diffusivity of the walls $i=1$ and $i=2$ should be contained in intervals:

$$
\begin{aligned}
& 0.545 \leq\left(a_{R} / a_{T}\right)_{i=1} \leq 0.630, \\
& 0.529 \leq\left(a_{R} / a_{T}\right)_{i=2} \leq 0.657 .
\end{aligned}
$$

\subsection{An attempt of applying the method to determine the characteristic features of the carbon/epoxy composite structure}

The course of action and the procedures used here were similar to those described in Section 3.2. To check the usefulness of the method for investigation of the transient heat exchange in multilayer composite materials, presented in this paper, the following experiment was carried out as below.

- Three samples were made by Polish company that produces composite materials: two of which were of the composite material consisting of respectively two and three layers of carbon fiber plate Porcher inside epoxy resin EP 53; the third sample was made of epoxy resin EP 53. All the samples had the same diameter and were comparable with respect of their thickness (Tab. 2).

- For the above samples measurements were made of the characteristic times $\tau_{R}$ and $\tau_{E, S, i}(i=2,3)$, and from relation (7) the equivalent thicknesses $l_{E, S, i}(i=2,3)$. The results of the measuring values of $\tau_{R}$ and $a_{R}$, as well as $\tau_{E, S, i}$ for $i=2,3$ are presented in Figs. 8 and 9 .

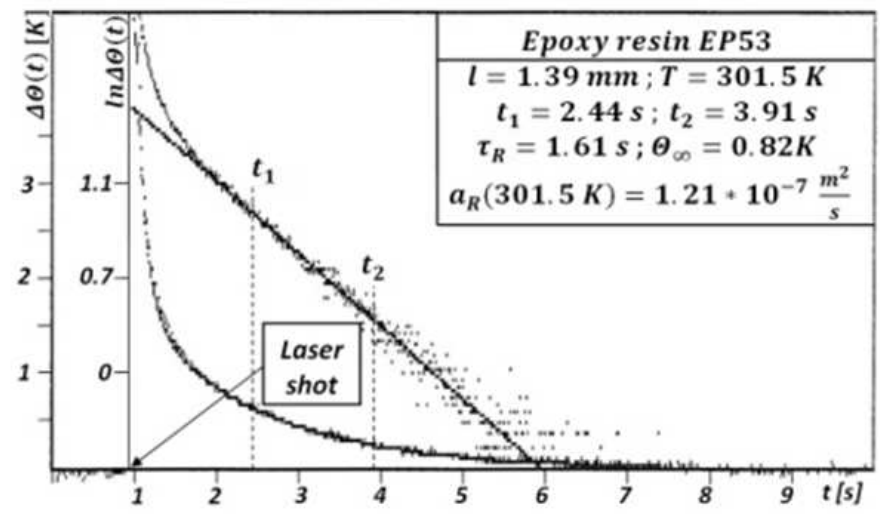

Figure 8: Distribution of experimental data during determination by the modified pulse method $\tau_{R}$ and $a_{R}$ for the epoxy resin EP53 at $T=301.5 \mathrm{~K}$. 

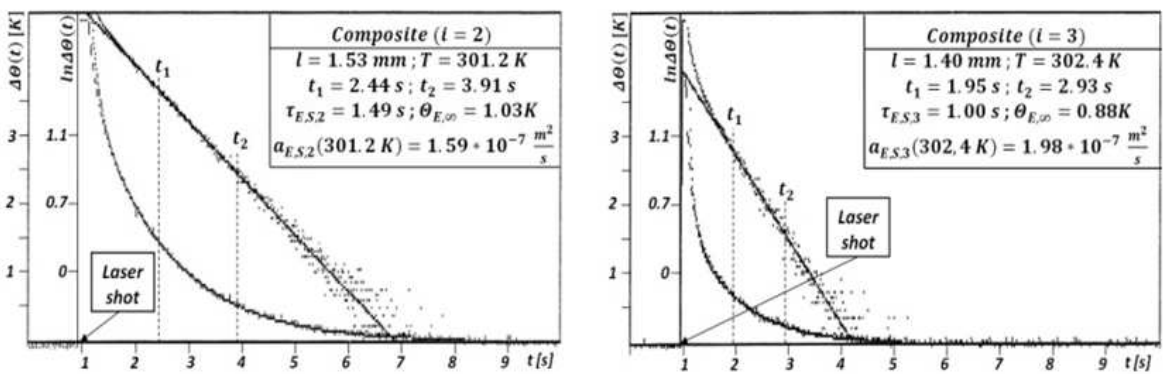

Figure 9: Distribution of experimental data during determination by the modified pulse method $\tau_{E, S, i}$ and $a_{E, S, i}$ for the carbon/epoxy composite sample with two and three layers $(i=2,3)$ of the carbon fiber plate.

- Then standardization of thicknesses of the samples was done in the way described in Section 3.2 and shown in Tab. 2. The thickness $l_{S}^{S}$, of the thickest sample was taken as the standard dimension thus $l_{S,}^{S}=$ $l_{S, 2}^{S}$. Table 2 summarises the post-standardization data (superscript ' $\mathrm{S}$ ').

- All fabrics used as reinforcement for a composite material have their own characteristic pattern of interweaving fibers, mostly carbon or glass fibers. In our case, the configuration of fiber bundles in the fabric is as shown in Fig. 10. Heat transfer in the carbon fiber fabric is a complex mechanism due to several factors including specific structure of carbon fiber fabric, a considerable difference between thermal diffusivity of epoxy $a_{R}$ and carbon fiber fabric $a_{C P, I I}$ along the axial direction of the fibers, and point interfacial areas between intersecting fibers. This leads to the reduction in the carbon fiber fabric thickness for transient heat transfer (Fig. 10). In the extreme case, the thickness can be reduced up to $50 \%$. In further calculations, however, the thickness of a single layer of Porcher 3692/120 carbon fiber fabric was taken to be $L=0.2 \mathrm{~mm}$.

- Based on the above results and the estimation of the non-dimensional carbon fiber fabric thickness $l_{C P, i} / l_{S}^{S}=i * L / l_{S}^{S}$ for $i=2,3$ in the composite, parameters $B_{i}^{S}(i=2,3)$ and the dimensionless values of the thermal diffusivities $\left(a_{R} / a_{C P}\right)_{i}$ for $i=2,3$ were calculated, using relation (9). The results of estimation are shown in Tab. 3 and Fig. 11. 
Table 2: The final result of standardization of epoxy resin EP53 and composite samples $(i=2,3)$ under investigation.
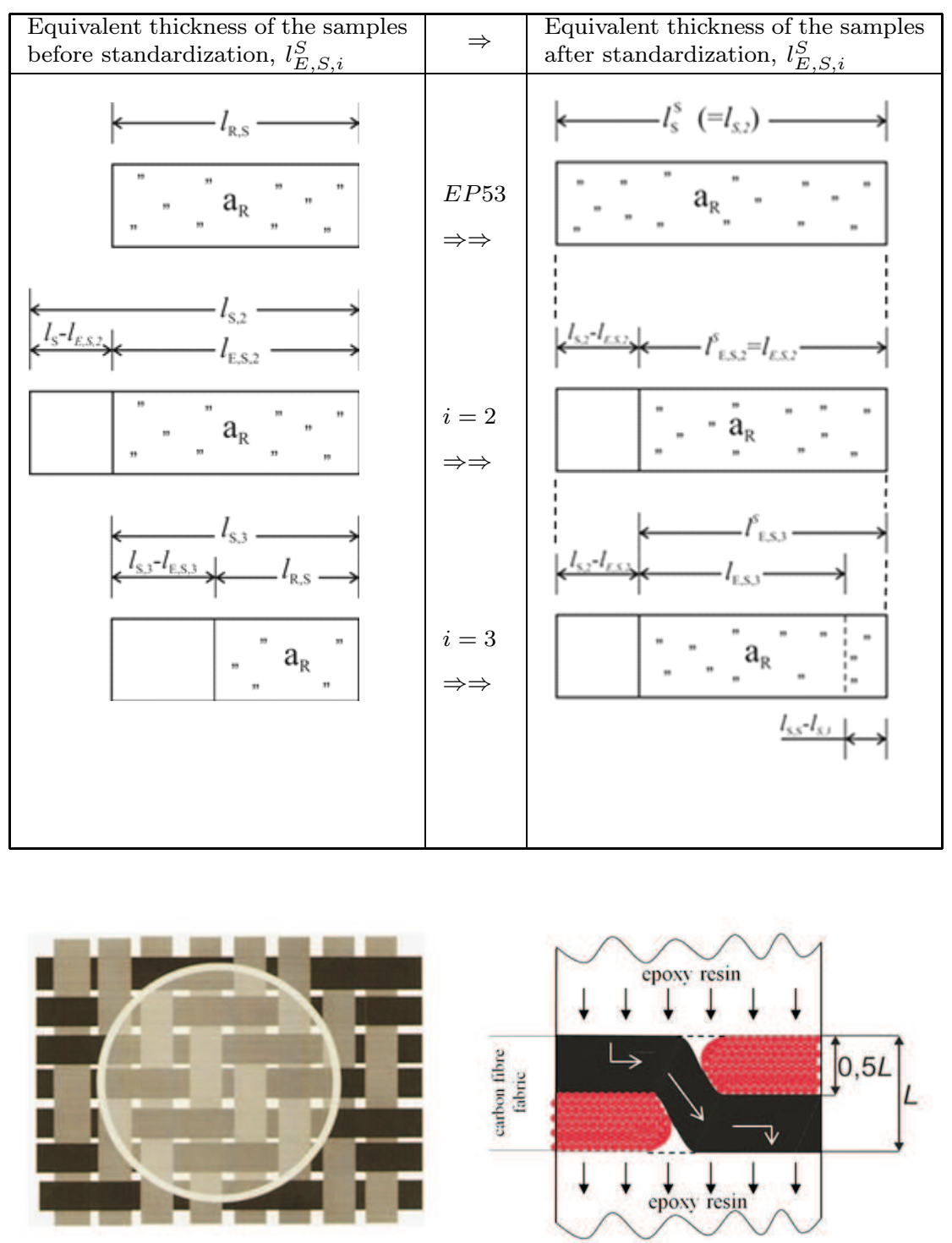

Figure 10: Configuration of interweaving bundles of carbon fiber in Porcher 3692/120 fabric with the marked of the investigation sample diameter $(12 \mathrm{~mm})$. 
Table 3: Results of the measurements and calculations for the epoxy resin EP53 and the two different carbon/epoxy composite samples $(i=2,3)$.

\begin{tabular}{|c|c|c|c|c|c|c|c|}
\hline Specification & $\begin{array}{l}\frac{l_{S, i}}{l_{S}^{S}} \\
{[\mathrm{~mm}]}\end{array}$ & $\begin{array}{c}\frac{\tau_{E, S}}{\tau_{E, S}^{S}} \\
{[\mathrm{~s}]}\end{array}$ & $\begin{array}{l}\frac{l_{E, S}}{l_{E, S}^{S}} \\
{[\mathrm{~mm}]}\end{array}$ & $\begin{array}{l}B_{i}^{S}= \\
l_{E, S}^{s} / l_{S}^{S}\end{array}$ & $\begin{array}{c}l_{C P} / l_{S}^{S} \\
l_{C P}= \\
0.2 * i \\
{[\mathrm{~mm}]}\end{array}$ & $a_{R} / a_{C P}$ & $a_{C P}$ \\
\hline $\begin{array}{ll}\text { Epoxy } & \text { resin } \\
\text { EP53*) } & \\
\end{array}$ & $\frac{1.39}{1.53}$ & $\frac{1.61}{1.772}$ & $\frac{1.39}{1.53}$ & 1.000 & 0 & - & - \\
\hline $\begin{array}{l}\text { Carbon/epoxy } \\
\text { composite - } \\
2 \text { layers }(\mathrm{i}=2) \\
\end{array}$ & $\frac{1.53}{1.53}$ & $\frac{1.49}{1.49}$ & $\frac{1.337}{1.337}$ & 0.874 & 0.261 & 0.268 & $3.73 a_{R}$ \\
\hline $\begin{array}{l}\text { Carbon/epoxy } \\
\text { composite - } \\
3 \text { layers }(\mathrm{i}=3)\end{array}$ & $\frac{1.40}{1.53}$ & $\frac{1.000}{1.194}$ & $\frac{1.0928}{1.2241}$ & 0.800 & 0.392 & 0.240 & $4.16 a_{R}$ \\
\hline
\end{tabular}

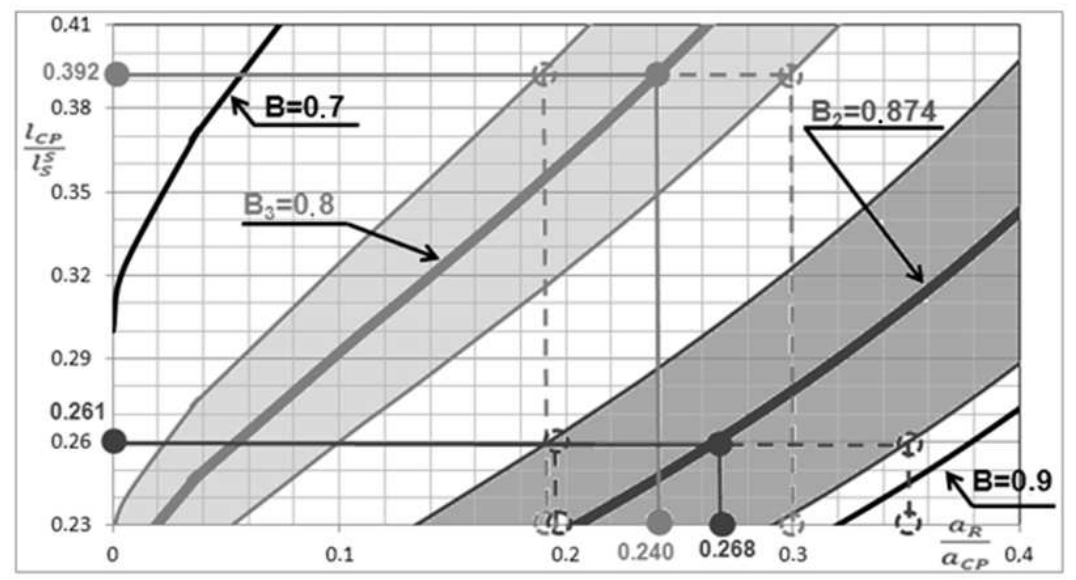

Figure 11: Graph of the function $\left(l_{C P, i} / l_{S}^{S}\right)_{B_{i}^{S}}=f\left[\left(a_{R} / a_{C P}\right)_{i}\right]$ for tested carbon/epoxy composite samples (see Tab. 3).

The effective thermal diffusivity values obtained for a single layer of the carbon fiber fabric of $L=0.2 \mathrm{~mm}$, in the direction transverse to the wall, i.e., $a_{C P}=3.73 a_{R}$ for the two-layer and $a_{C P}=4.16 a_{R}$ for the three-layer walls vary by less than $12 \%$.

Measurement errors were estimated according to the procedure described in Section 3.2 assuming that the single layer of carbon fiber fabric in the 
composite wall has the thickness of $L=0.2 \mathrm{~mm}$. For the samples with two and three layers of carbon fiber fabric, we have (Tab. 3, Fig. 11): $\left|\triangle a_{R} / a_{R}\right|=0.022,\left|\left(\triangle \tau_{E, S} / \tau_{E, S}\right)_{i=2}^{S}\right|=0.007,\left|\left(\triangle \tau_{E, S} / \tau_{E, S}\right)_{i=3}^{S}\right|=0.01$ and $\left|\triangle l_{S}^{S} / l_{S}^{S}\right|=0.007$. Substituting these values into (14) we obtain $\delta_{B, 2}=$ 0.02 and $\delta_{B, 3}=0.022$. The dimensionless values of the thermal diffusivity $\left(a_{R} / a_{C P}\right)_{i}$ of the walls $i=2$ and $i=3$ should be included in intervals:

$$
\begin{aligned}
& 0.194 \leq\left(a_{R} / a_{C P}\right)_{i=2} \leq 0.353, \\
& 0.188 \leq\left(a_{R} / a_{C P}\right)_{i=3} \leq 0.298 .
\end{aligned}
$$

Theoretically, with ideally manufactured composite samples, the following dependence should be satisfied

$$
\left(a_{R} / a_{C P}\right)_{i=2}=\left(a_{R} / a_{C P}\right)_{i=3} .
$$

In practice, however, condition (15) cannot be satisfied due to the following factors:

- it is impossible to place individual layers of carbon fiber fabric perfectly in parallel to each other and to the extreme surfaces of the samples under investigation;

- thermal resistances at the interface of the matrix and the carbon fiber fabric layers are difficult to be determined accurately and can hardly be accounted for separately;

- this also refers to a random distribution of thermal resistivity of the interface between single carbon fibers in the bundle and in-between the bundles of the fabric.

\section{Conclusion}

The paper presents an attempt to use the theory of similarity to investigate the transient heat exchange in materials with complex structure, in particular carbon/epoxy composites by developing a theoretical and experimental method for determining the equivalent value of thermal diffusivity for the wall from the known thermal diffusivities and thicknesses of layers forming a composite wall.

The proposed method was tested on two-layer composite sample 'epoxy 
resin EP53 - polyamide PA6'. The main idea in this test was to check whether the method proposed in the work of calculating thermal diffusivity value of polyamide PA6 is consistent with the value known from independent measurements. It was found that these values differ by less than $5 \%$, namely the error of measurement method.

Trying to apply the presented methods to analyze the characteristics of the composite wall structure 'carbon/epoxy' containing two and four layers of plates, showed that it can be used: for practical assessment of the correctness of theoretical assumptions and the proposed technology of composite walls; to estimate the value of thermal diffusivity of one of the layers of double-layer composite wall where their thicknesses and thermal diffusivity of the second component are known.

Tests of the transient heat transfer in the composite walls with two or three layers of Porcher 3692/120 carbon fabric have shown that a single layer of this fabric which is $L_{C P}=0.2 \mathrm{~mm}$ thick has the equivalent thermal diffusivity in a direction perpendicular to the sample surface equal to $a_{C P}=a_{C P, \perp} \cong 4 a_{R}=4.84 \times 10^{-7} \mathrm{~m}^{2} / \mathrm{s}$. This carbon fabric layer in a composite wall may be replaced by a matrix layer of EP53 epoxy resin with the thermal diffusivity $a_{R}$ and a thickness which is two time smaller, that is $L_{E, C P}=0.5 L_{C P}=0.1 \mathrm{~mm}$.

From the point of view of the heat transfer, the obtained results of the tests of the composite walls with two or three Porcher 3692/120 carbon fabric layers, seem to be credible when we take into account both the carbon fabric structure in which bundles of carbon fibers interweave each other at right angles, and the results of the tests of the carbon fibers' thermal diffusivity along and perpendicular to their axes $\left(a_{C P, \|} \cong 5 \times 10^{-6} \mathrm{~m}^{2} / \mathrm{s}-\right.$ own investigation, $a_{C P, \perp} \cong 4.84 \times 10^{-7} \mathrm{~m}^{2} / \mathrm{s}$ ). On their basis it may be concluded that each bundle is an integral adiabatic system in the fabric, by which the heat flux is transferred between the matrix layers of the fabric sticking on both sides to it.

Acknowledgments This work was supported by the Polish Ministry of Science and Higher Education under Research Project No. N N512 477639. 


\section{References}

[1] Carslaw H.S., Jaeger J.C.: Conduction of Heat in Solids, second edition. Oxford at the Clarendon Press 1959.

[2] Lienhard J.H. IV, Lienhard J.H. V: A Heat Transfer Textbook. Third Edition. MA Phlogiston Press Cambridge 2004.

[3] TerpiŁowski J.: A modified flash method for determination of thermal diffusivity in solids. Arch. Thermodyn. 24(2003), 1, 59-80.

[4] TerpiŁowski J.: A modified flash method for determination of thermal diffusivity in semitransparent solids exposed to laser radiation. Arch. Thermodyn. 25(2004), 2, $39-68$.

[5] Terpilowski J., Szczepaniak R., Woroniak G., Rudzki R.: Adaptation of the modified pulse method for determination of thermal diffusivity of solids in the vicinity of the second-order phase transition points. Arch. Thermodyn. 34(2013), 2, 71-90.

[6] Parker W.J., Jenkins R.J., Butler C.P., Аввot G.L.: A flash method of determining thermal diffusivity, heat capacity, and thermal conductivity. J. Appl. Phys. 32(1961), 1679-1684. 